\title{
الأضداد في اللغة المصرية القديمة
}

\section{The contronyms(opposites) in Ancient Egyptian language هبة رجب أبو بكر \\ مدرس اللغة المصرية القديمة - كلية الآثار - الأقصر}

\section{Heba Ragab Abu-Baker}

Lecturer of Ancient Egyptian Language - Faculty of Archeology - Luxor

hebaragab66@yahoo.com

تتتاول الورقة البحثية ظاهرة من ظواهر الاشتراك اللفظي في اللغة المصرية القديمة، وهي الأضداد ومفهومها في اللغة، ومدلول الضرب بين هذه الألفاظ من واقع النصوص، والتعرف على اللفظة الواحدة وأضدادها وصورها الكتابية المختلفة،

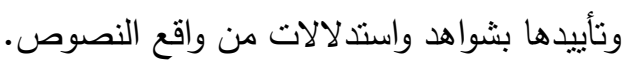

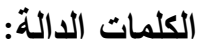

الأضداد؛ الاشتراك اللفظي؛ المدلول اللفظي؛ اللغة المصرية.

\section{Abstract:}

The research paper deals with a phenomenon of verbal association in the ancient Egyptian language, which is opposites and their concept in the language, and the meaning of multiplication between these words from the reality of the texts, and identifying the single word and its opposites and its various written images, and supporting it with evidence and inferences from the reality of the texts.

Key words:

Contronyms, Auto-antoym, Enantiosemy, Verbal participation, Opposites, Egyptian language. 


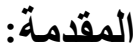

الأضداد في اللغة هي الألفاظ التي تقع على الثيء وضده في المعنى ،أي الكلمات التى تؤدى إلى

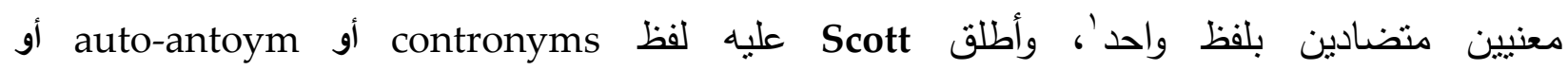
enantiosemy

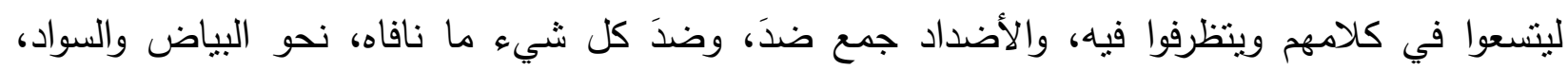

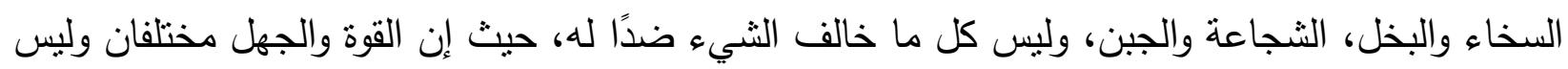

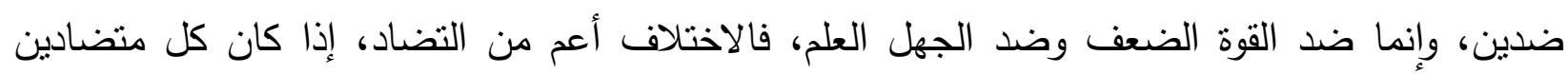
مختلفين وليس كل مختلفين متضادين".

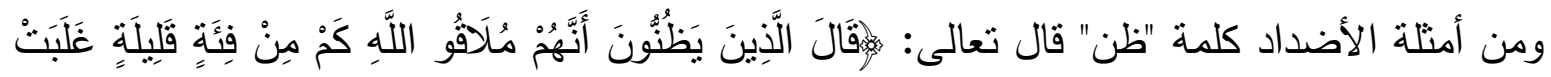

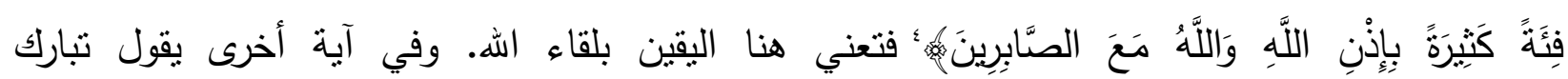

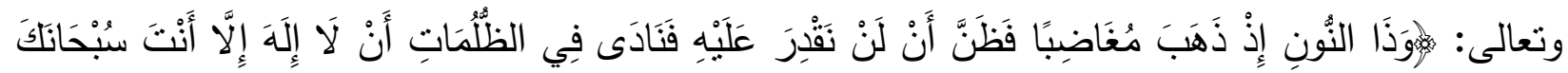

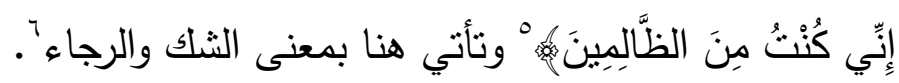

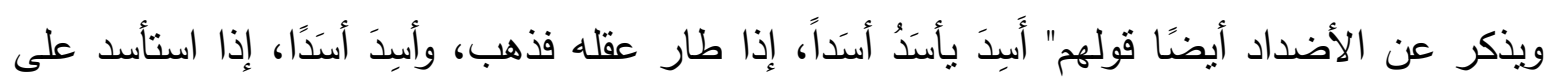

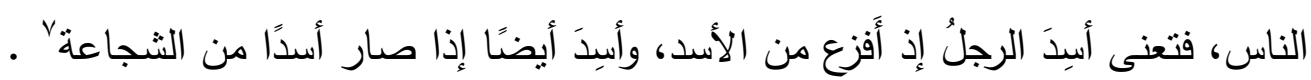

ويذكر أنطونيوس بطرس أن الأضداد قسم من المشترك اللفظي، و المشترك اللفظي لا يقع إلا في

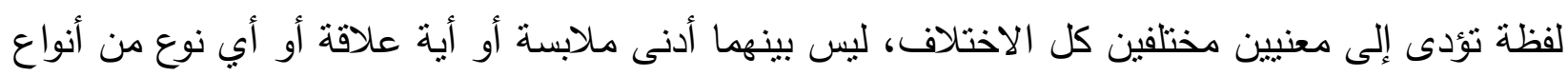

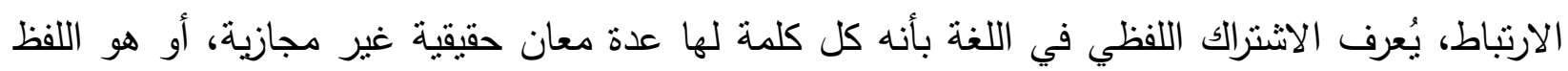

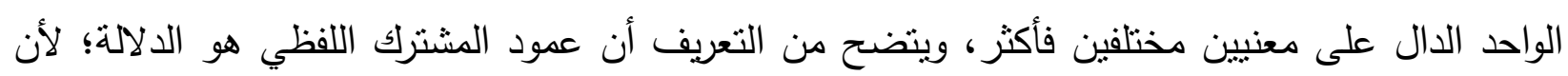

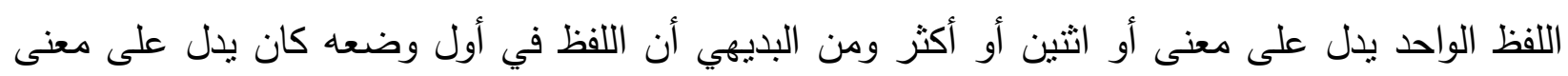

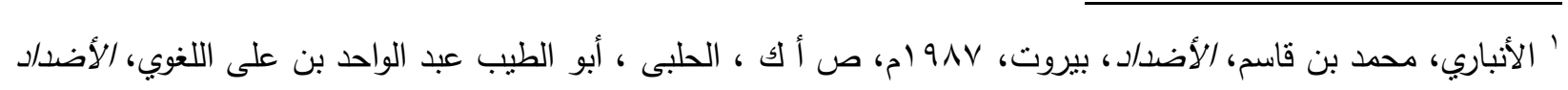

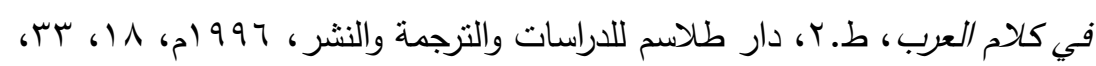
ScOTT, B.N., Wordplay in Ancient Near Eastern Texts, Ancient Near East Monographs 26, Atlanta, 2021,158. 2 SCOTT, Wordplay in Ancient Near Eastern Texts, 158.

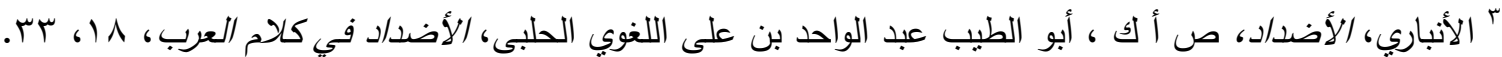

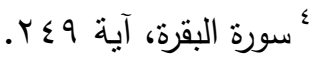

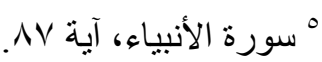

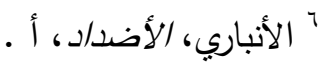

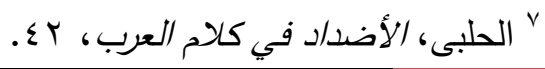


واحد ثم تولد من هذا المعنى عدة معانٍ ، وهذا التوالد هو ما نُسميه تطور المعنى، والتضاد هو أن يطلق اللفظ على المعنى وضده ، وهو إذا نوع من المشترك اللفظي ، فكل تضاد مشترك لفظي وليس العكس ^. ..... وسنتتبع هذه الظاهرة في اللغة المصرية القديمة ، وهل ثبت وجودها في اللغة، وسيتم عمل حصر لأغلب الألفاظ التي ينطبق عليها الضدية وعمل تحليل لمكنونات هذه الألفاظ ومدلولها السياقي:

يأتي هذا المصطلح بمعنى حمار ، حمير ، بليد ، بُلداء ، ومن صورها الكتابية :

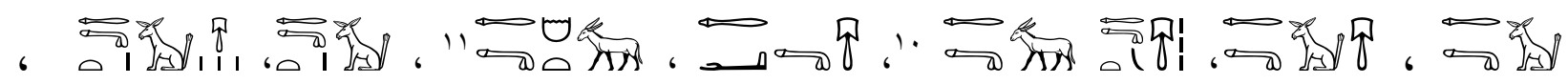

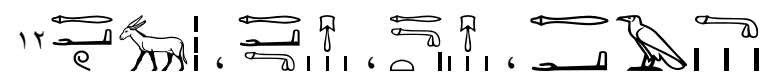

كما جاءت على عكس معناها فتعنى عظيم، نبيل، كبير "َ'، وتعددت صوره الكتابية على نحو السياق

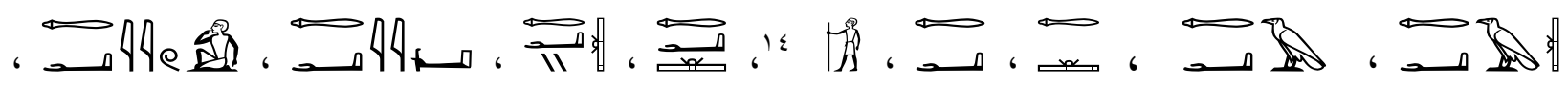

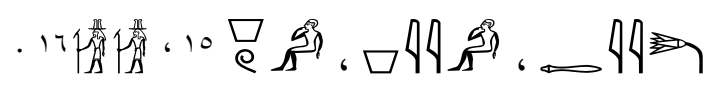

فقد ورد بسياق نص بردية القروى الفصيح الآتى : 几 1 a

h3t pw ir .n shty pn r kmt 3tp .n f $3 w . f$

هبط هذا الفلاح إلى مصر بعد أن حمل حميرهـ'.

^مكرم، عبدالعال سالم، المشترك اللفظي في الحقل القرآني، الكويت، طا، 997 (م، 9-0 1؛ بطرس، أنطونيوس، المعجم

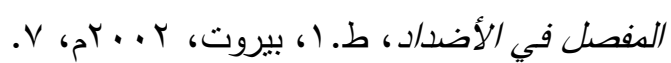

${ }^{9}$ ERMAN, \& GRAPOW, (EDS.): WB,,IV., 165,6; CD,38; ERMAN, \& GRAPOW, (EDS.): WB, B, , 140; BUDGE, I, 109.

${ }^{10}$ ERMAN, \& GRAPOW, (EDS.): WB, I., 165,6; CD,38; ERMAN, \& GRAPOW, (EDS.): WB, B, 140.

${ }_{11} \mathrm{CD}, 38$.

12 BUDGE, I, 109.

${ }^{13}$ ERMAN, \& GRAPOW, (EDS.): WB,,I., 161,3; CD,37; ERMAN, \& GRAPOW, (EDS.): WB, B, b, 137; BUDGE, I, 107.

${ }^{14}$ ERMAN, \& GRAPOW, (EDS.): WB,,I., 161,3; CD,37; ERMAN, \& GRAPOW, (EDS.): WB, B, , 137; BUDGE, I, 107

${ }^{15}$ BUDGE, I, 107.

${ }_{16}^{16}$ WPL, 133.

17 PARKInSON, R.B.,The Tale of the Eloquent Peasant, Oxford, 1991,R1.7; Allen, P.J., Middle Egyptian Literature, Cambridge, 2015, 232. R1,7.

^’حرفيًا: أنه الهبوط الذي فعله هذا الفلاح إلى مصر لأن الجملة كاملة ينطبق عليه صيغة f. f

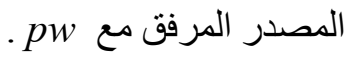




$$
\begin{aligned}
& \text { بينما ورد بالتعويذة رقم عV ب بنصوص الأهرم : }
\end{aligned}
$$

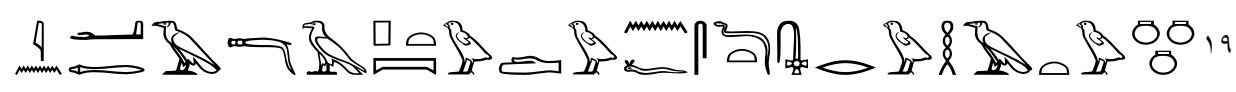

$$
\begin{aligned}
& \text { in } 3 \text { mhtiw pt wdw n f s } \underline{d} \text { t } r \text { wḩ } 3 w t
\end{aligned}
$$

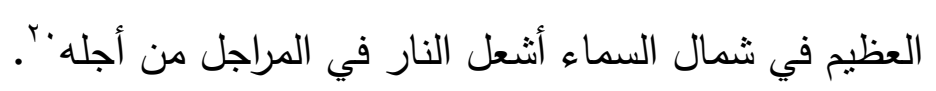

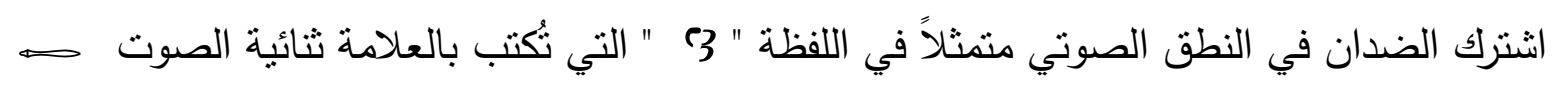

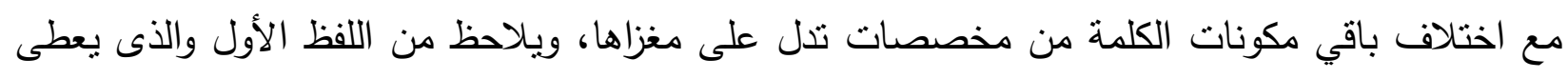
معنى حمار، وهذا اللفظ كرس وارتبط ولايزال مرتبط في الأذهان كأحد الثتائم المعبرة عن الغباء والتبلد نظرًا

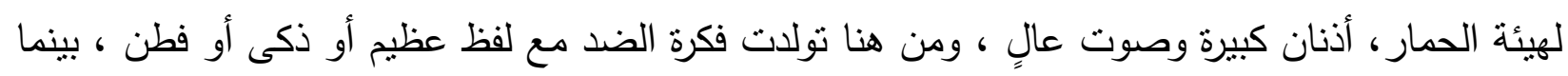

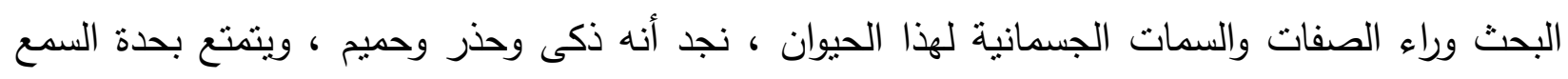

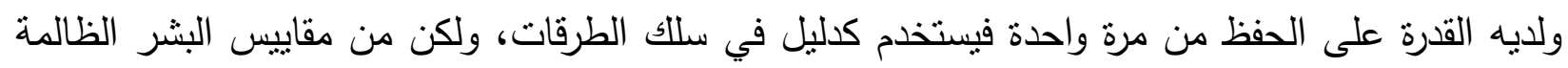

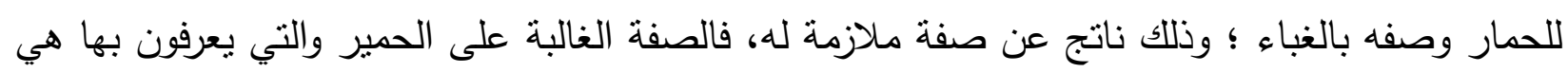

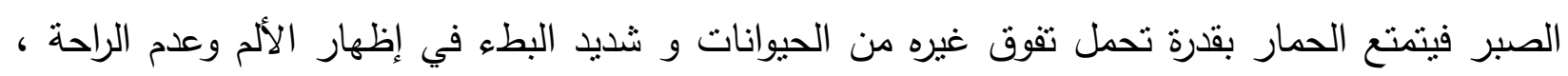

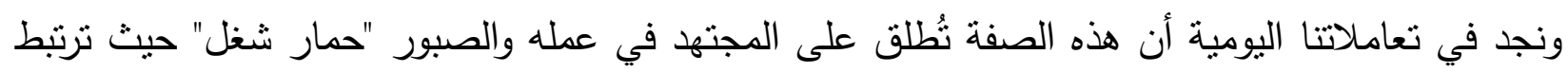

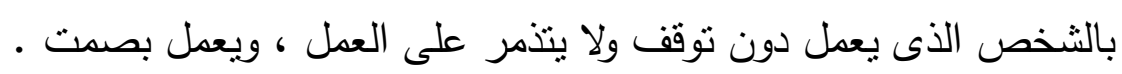
ولكن الباحث على يقين بأن المصري القديم لم يستخدم لفظ 3؟ للالالة على العظيم اعتباطًا وإنما

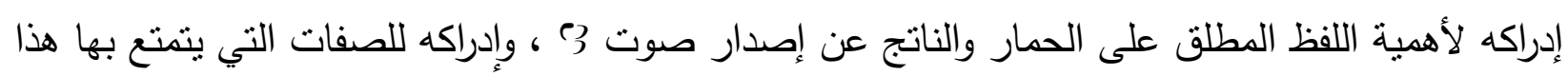

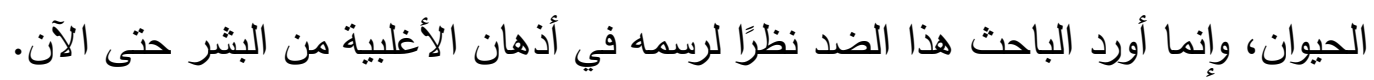
$b r$

جاء هذا اللفظ ليعنى عينان ، أبصر ، رأى ، شاهد ، يرى "، ومن صوره الكتابية :

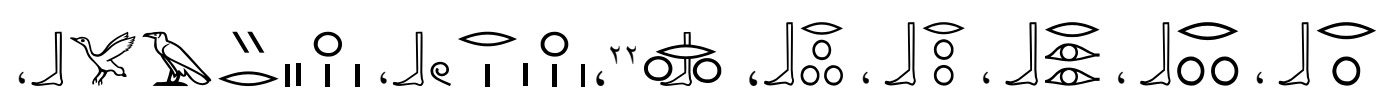

$$
\text { ris }
$$

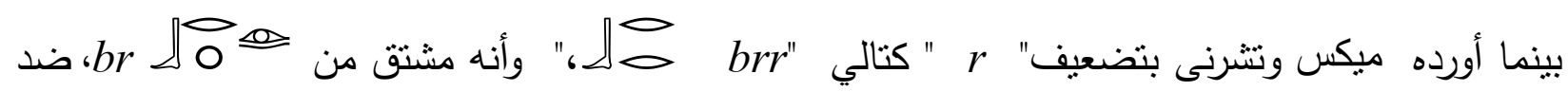

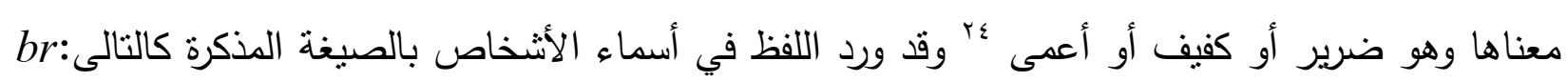

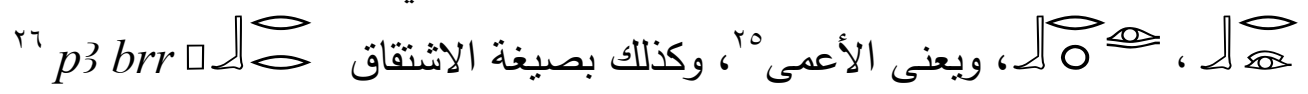

19 Pyr, 274.

${ }^{20}$ FAULKNER, Pyr,.81.

${ }^{21}$ ERMAN, \& GRAPOW, (EDS.): WB,,I., 465,5-7; ERMAN, \& GRAPOW, (EDS.): WB, B, , 273; BUDGE,I,219.

22 ERMAN, \& GRAPOW, (EDS.): WB,,I., 465,5-7; BUDGE,I,219 ; WPL, 322.

${ }^{23}$ ERMAN, \& GRAPOW, (EDS.): $W B, B, 273$. 
وهنا اشترك الضدان في نفس الصور الكتابية للكلمة ؛ نظرًا لأن الإبصار والإضرار يرتبط كلاهما بالعين فالمخصصات واحدة في هذا اللفظ .

ومن الممكن أن يكون الضد بين هذه الكلمات مجازى ،حيث نرى أن الشخص يكون بصيرًا ولكن بعض تصرفاته وعدم قدرته على اتخاذ القرارات تتم على أن يوصف من قبل أصحاب القرار بالأعمى، فنجد

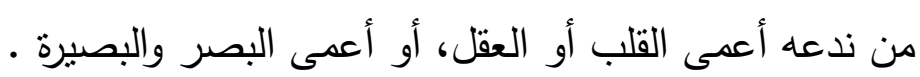

$: h b \cdot r$

ورد هذا المصطلح بمعنى عيد، مهرجان ، احتفال، نصر ب ، ومن صورها الكتابية :

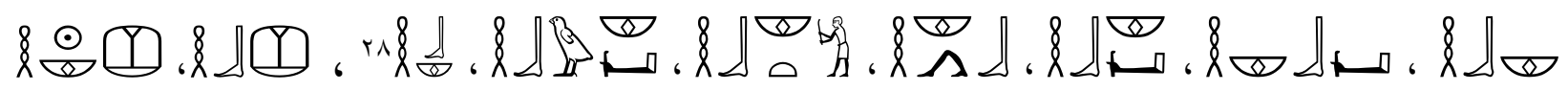
.

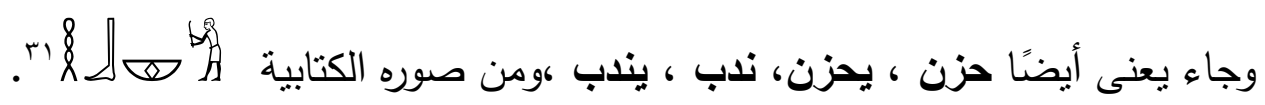
ورد اللفظ بمعنى احتفال بالتعويذة وس ا بنصوص التوابيت كالتالي:

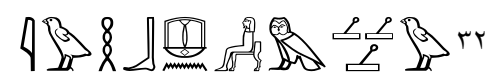
iw $h b . n$.i $m s 3 w$ احتقلت في بعض الأوقات "أحيانًا "rr" .

وورد بالسيرة الذاتية لحرخوف في تمنيات للعالم الآخر تمنلت في السياق التالي :

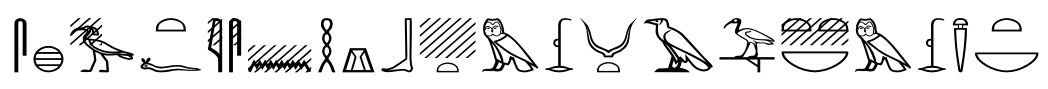

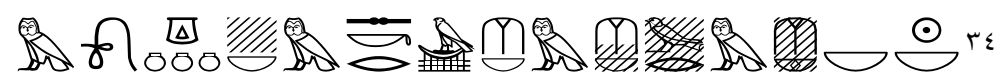

sḩ3t.f is n hri-ḥb m rnpt wp $m$ Dhhwti nb m rnpt tpit nbt $m$ w3g nb $m$ Skr ḥb nb m $h \bar{b} n b$ wr $m \underline{h b} n b-r^{c}$

\footnotetext{
${ }^{24}$ AnLex., No.77.1283, 120; ČERNY.I., Coptic Etymological Dictionary, New York, 1976, 23.

${ }_{25}$ PN.I, 97,27.

26 PN.I, 104,25.

${ }^{27}$ ERMAN, \& GRAPOW, (EDS.): WB,,III., 57,5; BUDGE, I, 474; LESKO, I, 307.

${ }^{28} \mathrm{~Wb}$, III., 57,5; BUDGE, I, 474; LESKO, I, 307.

${ }^{29}$ ERMAN, \& GRAPOW, (EDS.): WB, B,, 554.

${ }^{30} \mathrm{WPL}, 634$.

${ }^{31}$ ERMAN, \& GRAPOW, (EDS.): WB, III, 61,14; CD,167.

${ }^{32}$ CT II, spell 139, 173b.

${ }^{33}$ CARRIER.C., Textes des Sarcophages du Moyen Empier Egyptien, Tome I, ROCHER, 2004, spell 139, 173b, 329.

34 Urk I, 121,6-8.
} 


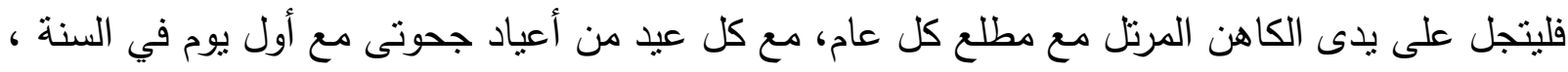

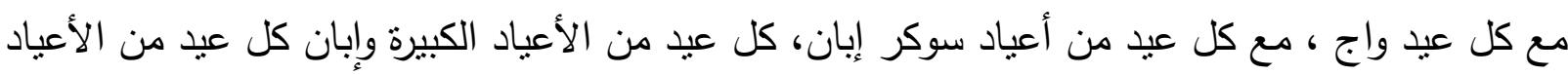
اليومية .

$$
\begin{aligned}
& \text { بينما ورد بنص سا نهت بمعنى حزن : }
\end{aligned}
$$

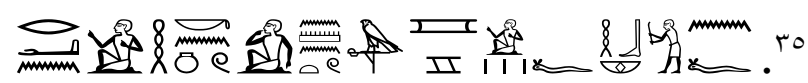

$$
\begin{aligned}
& \text { rdi n.f hlknw n mntw mrw f h hb n.f }
\end{aligned}
$$

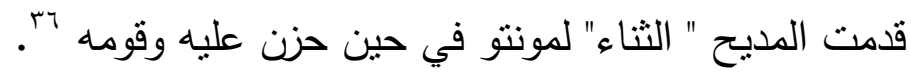

وردت hb لاى Blumenthal بمعنى حزن أو نعي ، بينما ذكرها Allen بمعنى ندب ، ووردت بتلك

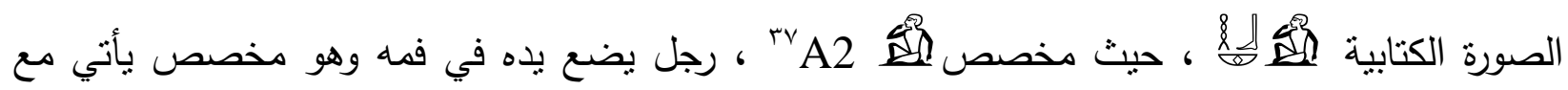
الكلمات ذات الدلالات المعبرة عن تحقيق رغبة ما ، الجوع، الثرب، الحديث، الصمت، والحب، وبهذا تلحق

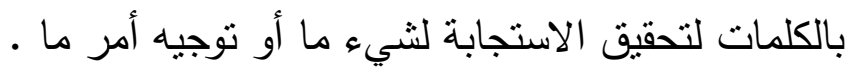
ويلاحظ انتراك الضدين للفظ hb في الصور الكتابية بما فيها من متممات و مخصصات، ويلاحظ

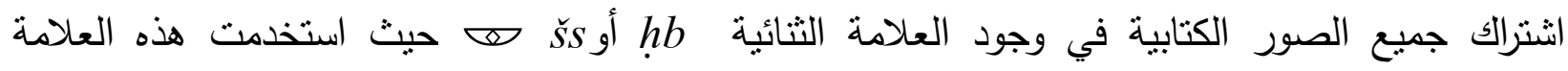

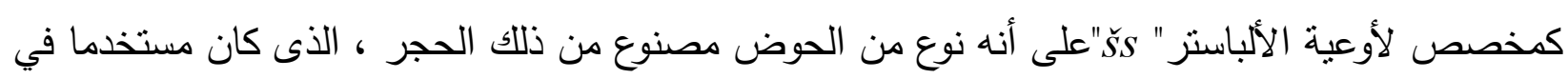

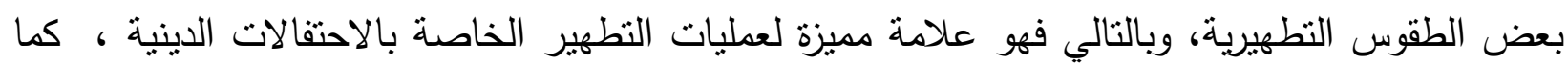

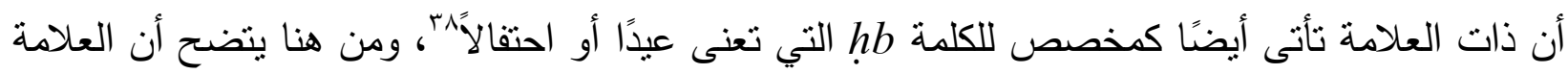
تأنى مخصصة للكلمات الدالة على احتفال سواء ديني أو جنائزي.

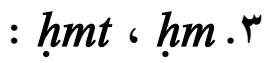

يعنى المصطلح جلاله ، فخامة " الملك" أو "الملكة" 9"ب ، وجاءت الكلمة لتعنى عكسها أيضًا بمعنى خادم، خادمة "“، ومن صورها الكتابية :

35 BlacKMAN, A.M., Middle Egyptian Stories, vol. II, Bruxelles, 1972, 28. B141-142.; KoCH, R., Die Erzählung des Sinuhe, Bruxelles, 1990, B141; ALLEN, P.J., Middle Egyptian Literature, 105. B141-142.

36 BlumENTHAL, E., Die Erzählung des Sinuhe, TUAT.III, Gütersloh, 1990-1997, 898;

ALLEN, P.J., Middle Egyptian Literature, 106. ${ }^{37}$ Eg.Gr, 442, A2.

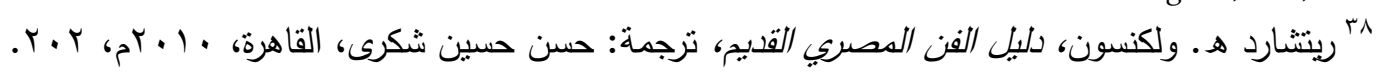

39 ERMAN, \& GRAPOW, (EDS.): WB,,III., 91,1; CD,169; ERMAN, \& GRAPOW, (EDS.): WB,B, 561; BUDGE, I, 482; LESKO, I, 310-311.

${ }^{40}$ ERMAN, \& GRAPOW, (EDS.): WB,,III., 87,13; CD,169; ERMAN, \& GRAPOW, (EDS.): WB, B, 562-567; BUDGE, I, 483; LESKO, I, 310-311; SCOTT, Wordplay in Ancient Near Eastern Texts, 158. 


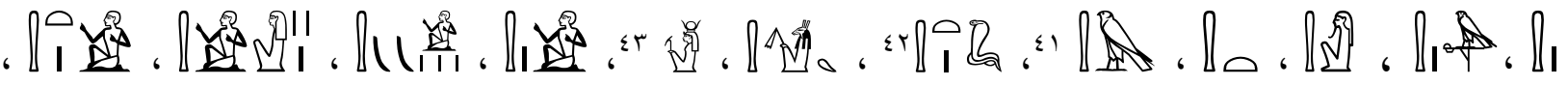

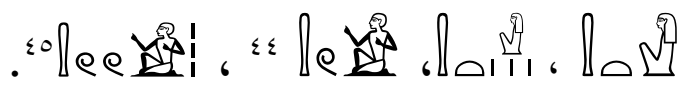
جاء بالسيرة الذاتية لأحمس بن إبانا على النحو التالى :

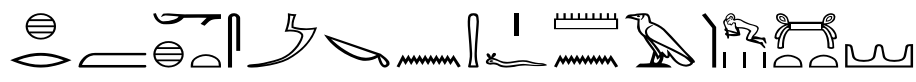

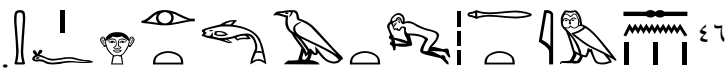

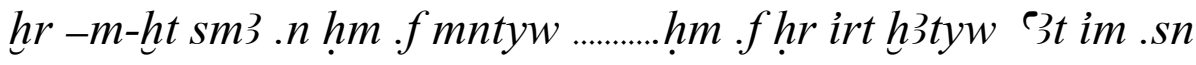

انظر الآن ذبح جالآته منتيو ....... بدا جلالته مذبحة كبيرة منهم

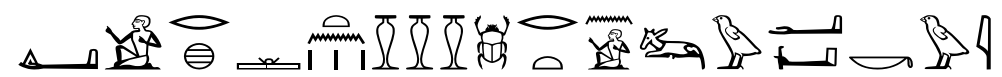

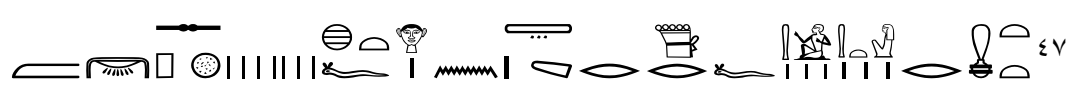

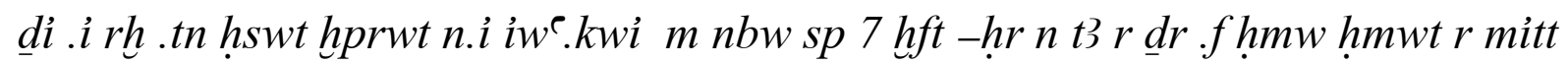
سأجعلكم تعرفون النعم الممنوحة لى، إنني كوفئت بالذهب سبع مرات أمام الأرض قاطبة ، والخدم والخادمات أيضًا

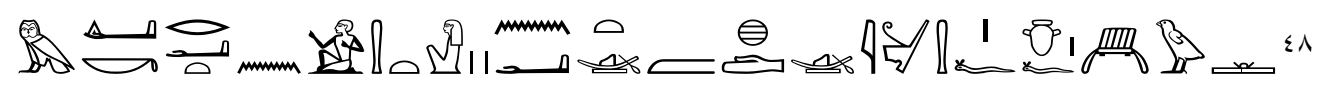

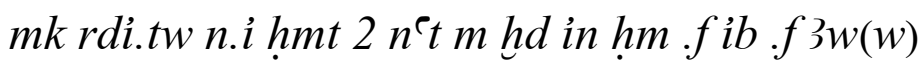

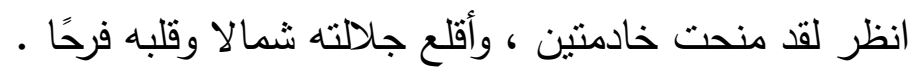

وأيضًا نقش ورد على قاعدة تمثال جالسًا القرفصاء " لباك ـ إن - خنسو "|؛ كبير كهنة آمون في

الكرنك والمشرف على الأعمال في معبد الأقصر في عهد الملك رعمسيس الثانى :

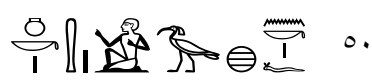

ink hm 3 h $n n b . f$

${ }^{41}$ CD,169; BUDGE, I, 474; LESKO, I, 310-311.

${ }^{42}$ ERMAN, \& GRAPOW, (EDS.): WB, $B, 567$.

${ }^{43}$ WPL, 643.

${ }^{44}$ CD,169; BUDGE, I, 474; LESKO,I, 310-311.

${ }^{45} \mathrm{WPL}, 643$.

${ }^{4}$ Urk IV, 5,4-14.

47 Urk $I V, 2,1-3$.

${ }^{4}$ Urk IV,5,4-14.

. PM, I, 61

${ }^{50} \mathrm{KRI}$, III, 297,11. 
أنا الخادم النافع لسيده

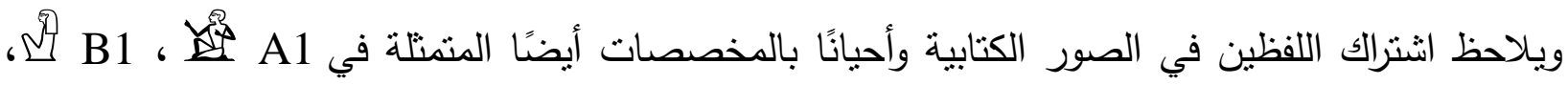

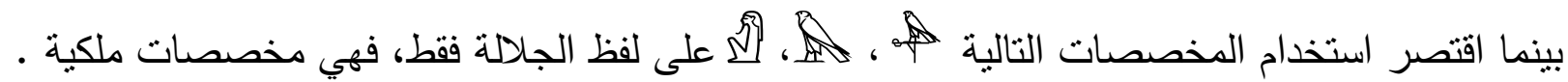

$: \underline{h w s} \cdot \varepsilon$

هذا اللفظ يعنى يينى، يؤسس، يشبد، يعزز ، يخطط ، يُركب ، يُرتب، يقيم ، وجاء اللفظ يعنى عكس ما ذكر من مصطلحات أيضًا تمثل في يسحق، يضرب، يقصف، يدمر ، يقتل بهّ، ومن صورها الكتابية :

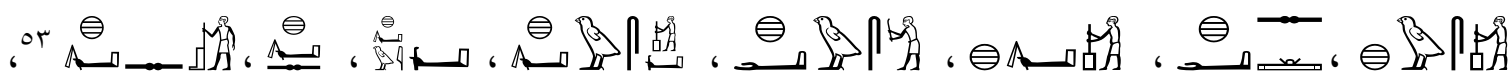

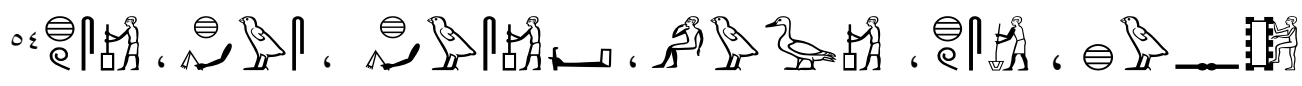

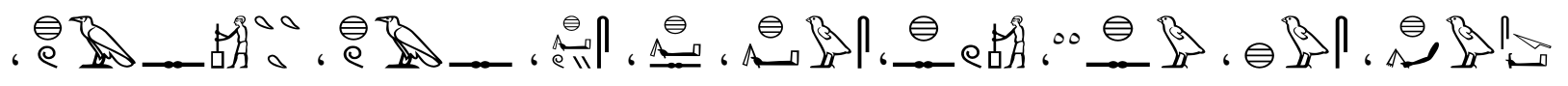

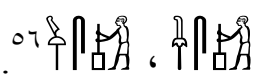

ويلاحظ اشتراك الضدين في معظم الصور الكتابية في المخصص A34 وهو يمثل رجل يسحق بأداة معينة أو بالهاون ويطن وورد بنص سانهت : (1)

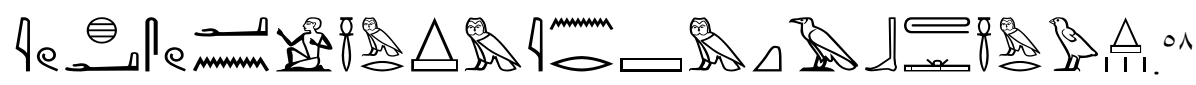

iw ḩwsw n.i mr m inr $m$ ḳ̉b mrw نُصب " شُيُ " لي هرم من الحجر وسط الأهرامات 9هـ

وورد نفس اللفظ بالضد بنص السيرة الذاتية الخاصة بالوزير رخمي رع في السياق التالي:

${ }^{51}$ KRIT, III, 213;

كلير لالويت، نصوص مقسة ونصوص دنيوية من مصر القديمة، ج. ا، ترجمة ماهر جويجاتى، مراجعة: طاهر عبد الحكيم،

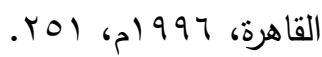

52 ERMAN, \& GRAPOW, (EDS.): WB,III, 248,4,16; CD,186; ERMAN, \& GRAPOW, (EDS.): WB,B,, 633; Budge,I,538; Lesko.I,354.

${ }^{53}$ ERMAN, \& GRAPOW, (EDS.): WB, III.,248,4,16; CD,186;HWb; 633; BUDGE,I,538; LESKO, I, 354.

54 ERMAN, \& GRAPOW, (EDS.): WB,III.,248,4,16; CD,186; BUDGE, I, 538; LESKO, I,354, WPL, 712.

${ }^{55}$ ERMAN, \& GRAPOW, (EDS.): WBIII.,248,4,16; CD,186; ERMAN, \& GRAPOW, (EDS.): WB; 633; BUDGE, I, 538; LESKO, I, 354.

${ }^{56}$ ERMAN, \& GRAPOW, (EDS.): WB, III,248, 4,16; CD,186; BUDGE, I, 538; LESKO, I,354, WPL, 712.

${ }^{57}$ Eg.Gr, 446.

${ }^{58}$ BlaCKMAN, Middle Egyptian, B300; KoCH, Die Erzählung des Sinuhe, B300; Allen, J.P., Middle Egyptian Literature, 150, B300.

${ }^{59}$ Blumenthal, Die Erzählung des Sinuhe, 910. 


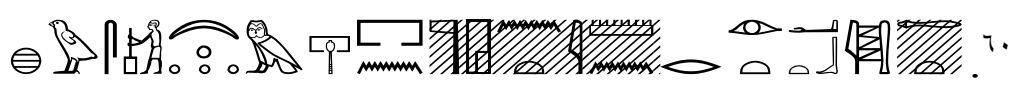

$\underline{h} w s$ w $h$ m pr-ḥd $n$ ḥwt-ntr nt'Imn

سحق" طحن" بذور الخروب من خزبنة معبد آمون لعمل كومة قرابين

: $h n p$.

يأتي هذا اللفظ بمعنى يسرق، يسلب ، ينهب ، جشع ، ومن صورها الكتابية :

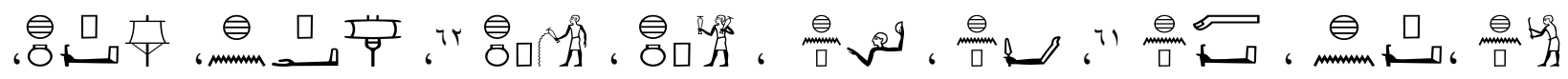
reo

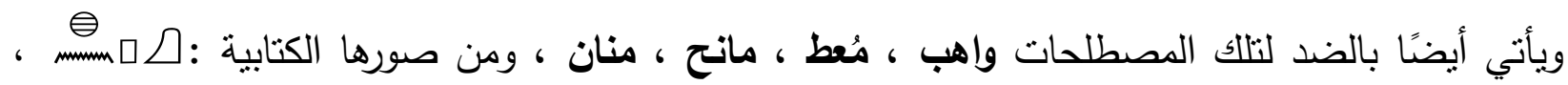

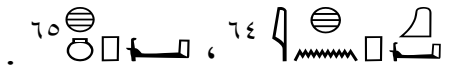
ونتطرق للأدلة من واقع نصوص الأهرام والتوابيت من خلال التالي: d

Wsr N nit n.k irt ḥr $n$ ḥmt $n m^{\odot}$ stš h̆hp $n . f . s$

أوزير خذ عين حور التي أنقذتها من ست عندما انتز عها "حرفيا سرقها " VI

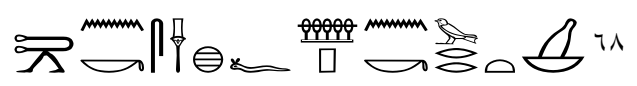

itî $n . k$ shm f. hnnp $n . k$ wrrt ff

خذ صولجانه وتلق حرفيا " امنح نفسك " ناج ورر 79.

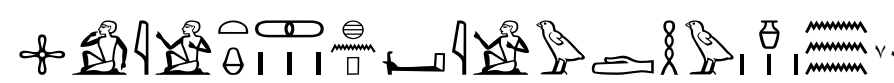

Wnm .i thnp .i wdhw

60 Urk IV, 1141, 2-3.

${ }^{61}$ ERMAN, \& GRAPOW, (EDS.): WB,III., 290,6,11; ERMAN, \& GRAPOW, (EDS.): WB, B, 648.

62 ERMAN, \& GRAPOW, (EDS.): WB,III., 290,6,11.

${ }^{63}$ WPL, 732.

${ }^{64}$ ERMAN, \& GRAPOW, (EDS.): WB,III., 290,15,19.

${ }^{65}$ WPL, 732.

66 Pyr, 652.

${ }^{67}$ FAULKNER, Pyr, 268.

68 Pyr, 677.

${ }^{69}$ FAULKNER, Pyr, .291.

${ }^{70}$ CT II, spell 149, 253c. 


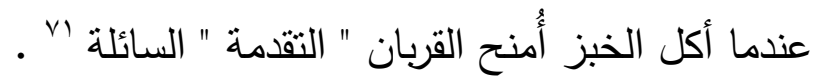

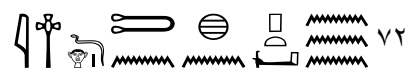

\section{ind $\underline{h r}$.th h hnpt $m w$}

$$
\text { سلامًا على مانح الماء }
$$

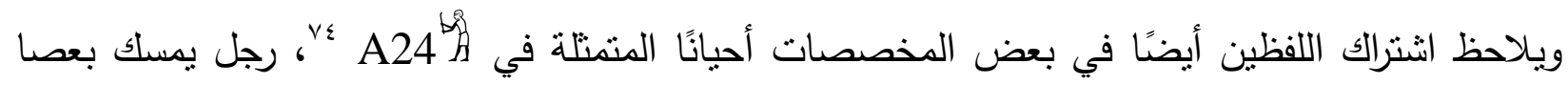
وهى تعبر عن العنف والثدة وكذلك تعبر عن الرغبة الثديدة في تتفيذ المبتغى ، وكذلك Z Z9 وتمنل عصاتين متقاطعتين وهى مخصص مناسب وملائم لبعض المعاني؛ لأن المصري القديم أدرج هذا المخصص لاستخدامه في بعض الكلمات المعبرة عن الضرر أو الأذى منل يكسر أو يخرب ، وكذلك المعاني الدالة على الاستجابة مثل يجيب أو يهُم، ومن هنا يظهر الضد في بعض الكلمات المستخدم فيها

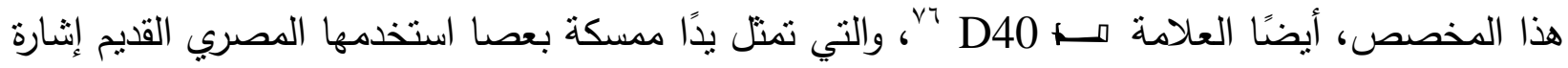
للعنف وكذلك أحيانا للنصح وتحقيق الاستجابة .

: šin. 7

يعنى هذا المصطلح يبطئ، يتأخر، ينتظر مV كما أنه يأتي أيضًا بألفاظ عكس ما ذكر ومنها جرى، إندفع، ركض، يستعجل، يتعجل الخطوة ، يتحرك ، يتقدم بسرعة لم، ومن صوره الكتابية : . 14 死

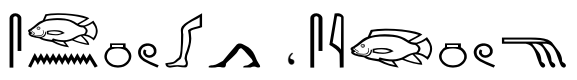
، .

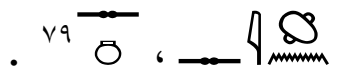

ويلاحظ اشتراك اللفظين في معظم المخصصات الموضحة لبيان ماهية اللفظ المتمنلة في دـ D54 التي يقع على عاتقها مسؤولية الحركة أو الوقوف .

${ }^{71}$ FAULKNER, CT, 128; CARRIER, Textes des Sarcophages, spell 149, 253c, 363.

${ }^{72}$ CT VI, spell 598, 215b.

${ }^{73}$ FAULKNER, CT, 193; CARRIER, Textes des Sarcophages, Tome II, spell 598, 215b, 1393.

${ }^{74} \mathrm{Eg}$.Gr, 444.

${ }^{75} \mathrm{Eg} . \mathrm{Gr}, 538$.

${ }^{76}$ Eg.Gr, 445.

77 ERMAN, \& GRAPOW, (EDS.): WB,IV., 38,4,8; CD,213; ERMAN, \& GRAPOW, (EDS.): WB,B,. 722; LESKO, II,12.

${ }^{78}$ ERMAN, \& GRAPOW, (EDS.): WB,IV., 38,9,20; CD,213; ERMAN, \& GRAPOW, (EDS.): WB, B, 722; BUDGE, II, 643.

79 ERMAN, \& GRAPOW, (EDS.): WB,IV, 38,4,8; CD,213; ERMAN, \& GRAPOW, (EDS.): WB,B, 722; LESKO, II, 12; BUDGE, II, 643. 


$$
\begin{aligned}
& \text { وسيتضح أمر الضدين للفظ من واقع السياقات التالية : }
\end{aligned}
$$

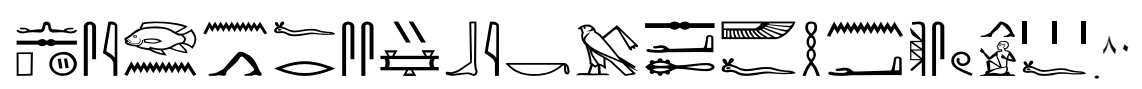

$$
\begin{aligned}
& n \text {-sp sinn frssi bik } \complement \underline{h} . f h n^{\complement} \check{s} m s w . f \\
& \text { لم يتردد لحظة " بتباطأ" ، طار الصقر مع أتباعه' ل. }
\end{aligned}
$$

الفعل sinn كتب بتضعيف الـ n قبل المخصص، ويذكر Gardiner,Allen أن sinn على ما يبدو اشتقت من الجزع والذى يعنى تأخير أو تباطؤ ، تردد .

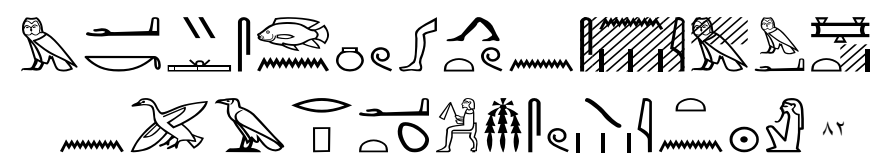

يركض الذى بين الصقور من أجل كا الملكء^.

80 GARDINER, A.H., Notes on The Story of Sinuhe, Paris, 1916, 11; BLACKMAN, Middle Egyptian, R20-21; KOCH., Die Erzählung des Sinuhe,, R20-21; ALLEN, Middle Egyptian Literature, 61R20-21.

81 BluMENTHAL, Die Erzählung des Sinuhe, 888.

82 pap.AnI , 15, 6, Gardiner, H.A., Egyptian Heratic Texts, part I, Leipzig, 1911, 27.

83 GARDINER, Egyptian Heratic Texts, 17-18.

84 Pyr, 602,1672 a.

${ }^{85}$ FAULKNER, Pyr, 248. 


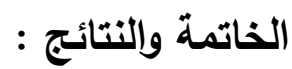

\section{يتضح من دراسة هذه الورقة البحثية التالي :}

- ورود الاشتراك اللفظي عامة وظاهرة الأضداد خاصة في معظم المراحل اللغوية.

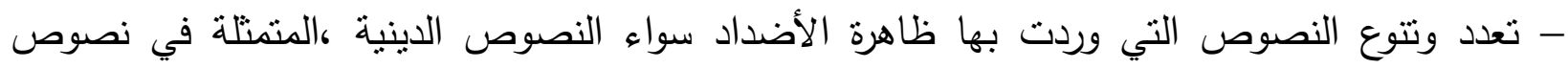
الأهرام أو نصوص النوابيت، وكذلك كتاب الموتى، النصوص الأدبية والسير الذاتية والنقوش الأثرية .

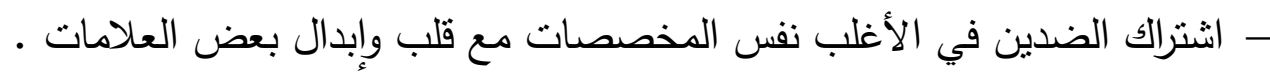
- تدل الألفاظ التي تحمل معنيين متضادين في اللغة المصرية القديمة على عبقرية اللغة في إعطاء الألفاظ

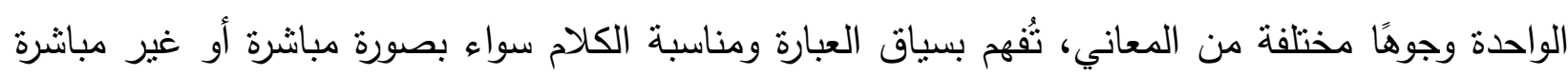

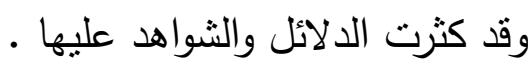
- وجود ظاهرة الأضداد في اللغة المصرية القديمة ناتج عن التطور في الاستعمال ونتيجة الجديد في لإني

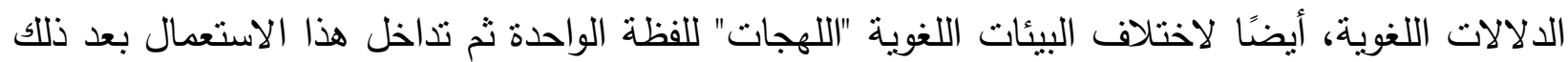

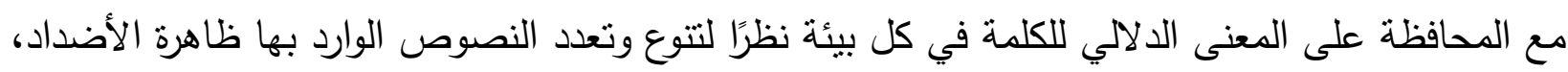
حيث إن أصحاب نلاك النصوص من فترات تاريخية مختلفة وكذللك بيئات مختلفة، كما في وفتنا الحالي اختلاف في اللهجات ، حيث توجد مصطلحات في اللغة العربية تذكرها إحدى البلدان بمعنى، ويظهر نفس بله

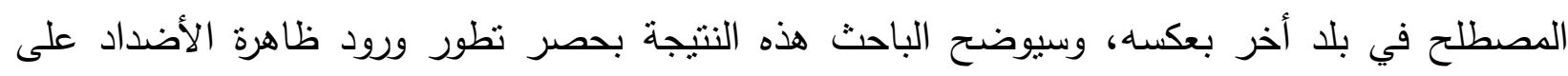

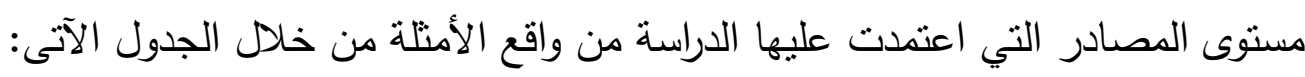




\begin{tabular}{|c|c|c|c|}
\hline \multicolumn{3}{|c|}{ مصادر الأضداد } & \multirow{2}{*}{ اللفظ وأضداده } \\
\hline النقوش الأثرية & النصوص الأدبية & النصوص الدينية & \\
\hline & نص القروى الفصيح & نصوص الأهرام & 3 \\
\hline سبرة حرخوف & نص سانهت & نصوص التوابيت & $h b$ \\
\hline خ و قاعدة أحمس بن إبانا & & والتوابيت وكتاب الموتى الأهرام & $h m-h m m t$ \\
\hline \multirow[t]{2}{*}{ سيرة رخمي رع } & نص سانهت & $\longrightarrow$ & $h w s$ \\
\hline & & نصوص الأهرام & hnnp \\
\hline- & نص سانهت & نصوص الأهرام & $\sin$ \\
\hline
\end{tabular}




\section{ثبت المصادر المراجع}

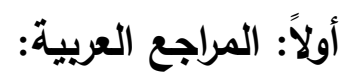

- The Holy Quran.

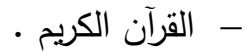

- الحلبى، أبو الطيب عبد الواحد بن على اللغوي، الأضد/د في كلام العرب، ط.r، دار طلاسم للدراسات والترجمة والنشر، .01997

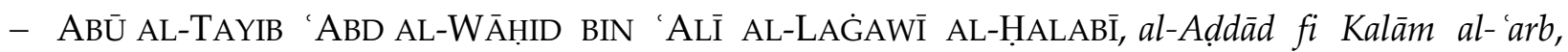
2. nd ed, Dār Ṭalāsim li'1-Dirāsāt wa'1 -tarğma wa'1- Našr,1996.

$$
\text { - بطرس، أنطونيوس، الدعجم المفصل في الأضد/د، ط. ا، بيروت، r . . rم. }
$$

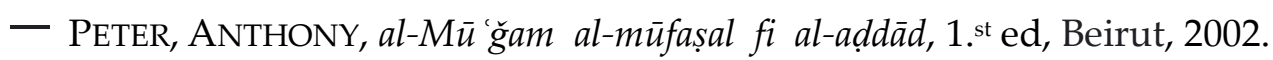

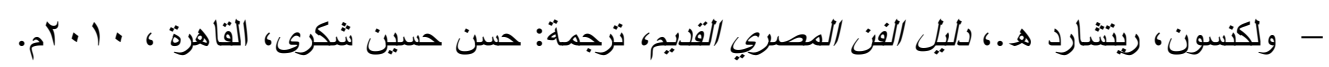

- WILKINSON, RiCHARD H., Dalīl al-fan al-miṣrī al-Qadīm, translated by :Ḥasan Ḥūsīn Šūkrī, Cairo, 2010.

$$
\text { - مكرم، عبدالعال سالم، المشترك اللفظي في الحقل القرآني، ط. ا، الكويت، } 999 \text { ام. }
$$

- 'ABD AL-'Al SĀLIM MAKRAM, āl-Mūštark al-lafẓ̂̀ fi al-Haql āl-Qūrānī, 1.st ed, Kuwait,1996.

- لالويت، كلير، نصوص مقدسة ونصوص دنبوبية من مصر القدبية، ج. ا، ترجمة ماهر جويجاتى، مراجعة: طاهر عبد

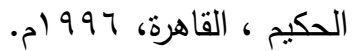

- Lallwett, Claire, Nūṣūṣ mūqdasa wa nūṣ̄ụș Dūnīwìya min Miṣr al-Qadìma, vol .1,translated by: Māhir Ğwyğāty, Reviewed by: Tāhir 'Abd alHakīm,Cairo,1996.

$$
\text { - الأنباري، محمد بن قاسم، الأضد/د، بيروت، } 9 \text { أم. }
$$

- AL-'ANBĀRĪ, MŪHAMMAd BIN QĀSIM, al-Aḍdad, Beirut, 1987.

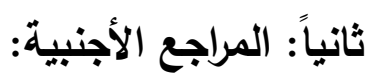

- AlLEN, J.P., Middle Egyptian Literature, Cambridge, 2015.

- BlackMAN., A.M., Middle Egyptian Stories, vol. II, Bruxelles,1972.

- BlumentHAl, E., Die Erzählung des Sinuhe, TUAT.III. Gütersloh, 1990-1997,

- Budge,W., An Egyptian Hieroglyphic Dictionary, Vol.I-II, London, 1920.

- CARRIER, C., Textes des Sarcophages du Moyen Empier Egyptien, Tome I, Rocher, 2004.

- ČERNY, I., Coptic Etymological Dictionary, New York, 1976.

- DE BuCK, A., The Egyptian Coffin Texts, 7 Vols, Chicago, 1935-1961.

- ERMAN, A. \& GRAPOW, H. (eds.): Worterbuch der Agyptischen Sprache V, Leipzig (J. HINRICHS) 1926 - 1931 [= WB]FAULKNER, R.O., The Ancient Egyptian Pyramid Texts, Oxford, 1969.

- FAULKNER, R.O., The Ancient Egyptian Coffin Texts, 3 Vols, London, 1973-1977.

- ...................., A Concise Dictionary of Middle Egyptian, Oxford, 2017.

- GARDINER, A.H., Egyptian Grammar, 3 ${ }^{\text {rd }}$ edition, London, 1973.

- .................., Egyptian Heratic Text, Leipzig, 1911.

- ..............., Notes on The Story of Sinuhe, Paris, 1916. 
- HANNIG, R., Die Sprche der Pharaonen Grosse Handwörterbuch, Ägyptisch -Deutsch (2800-950 v-Chr), Germany, 2005.

- HELCK, W., Urkunden der 18.Dynastie, Berlin, 1958.

- KITCHEN, K.A., Ramesside Inscriptions Historical and Biographical, Vols.1,-,5, Oxford, 1975-1983.

- ..............., Ramesside Inscriptions Translated and Annotated :Translations, Vols.1,-,5, Oxford, 19932008 .

- KOCH., R., Die Erzählung des Sinuhe, Bruxelles, 1990.

- LESKO, L.H., A Dictionary of Late Egyptian, 2 Vols, USA, 2002 - 2004.

- MeEKS, D., Année Lexicographique, 3 Tomes, 1977-1979, Paris, 1980-1982.

- PARKINSON, R.B ., The Tale of the Eloquent Peasant, Oxford, 1991.

- Porter, B \& Moss, R.L.B., Topographical Bibliography of Ancient Egyptian Hieroglyphic Texts , Reliefs, and Paintings, Vols .7, Oxford, 1934-1995.

- RANKE .H., Die Agyptischen Personennamen, 3 bands, Gluckstade Hambourg, New York, Berlin, 19351976.

- SETHE, k., Urkunden der 18. Dynastie, Leipzig, 1906.

- ..........., Urkunden des Alten Reichs, Leipzig, 1933.

- ..........., Die Altagyptisch Pyramidentexte, 2 Bande, Hildesheim, 1960.

- Scotт B.N., Wordplay in Ancient Near Eastern Texts, Ancient Near East Monographs 26, Atlanta, 2021, 158.

- WiLsON, P., Aptolemaic Lexikon: A lexicographical Study of the Texts in the Temple of Edfu, Leuven, 1997. 